Von Heyl, J. and Demir, S.-T. (2019). "Digitizing Lean Construction with Building Information Modeling." In: Proc. $27^{\text {th }}$ Annual Conference of the International. Group for Lean Construction (IGLC), Pasquire C. and Hamzeh F.R. (ed.), Dublin, Ireland, pp. 843-852. DOI: https://doi.org/10.24928/2019/0122. Available at: <www.iglc.net>.

\title{
DIGITIZING LEAN CONSTRUCTION WITH BUILDING INFORMATION MODELING
}

\author{
Jakob von Heyl ${ }^{1}$ and Selim-Tugra Demir ${ }^{2}$
}

\begin{abstract}
Construction projects are becoming more complex due to tighter project schedules, increasing cost and quality pressures and associated continuous change during the execution phase. Technical requirements are also increasing, and division of work between the various technical experts involved is highly fragmented. This results in construction projects with unreliable schedules, cost overruns and defects resulting from the lack of coordination on site. Lean Construction Management (LCM) and Building Information Modelling (BIM) are possible solutions to these challenges. In the past, these two methodological approaches have normally been used independently of each other. The combined implementation of the two methodologies requires the highly systematic setup of project structures, processes and data. This results in huge demand for computing and automation solutions to facilitate and support this synthesis. This paper reports on the implementation of a digital LCM approach. The use of QR codes, Kanban cards and daily meetings demonstrates a new platform to add value to the BIM model and to construction site collaboration. Case study data from five pilot projects - as well as images and workshop findings - are presented and discussed, and the successes achieved and the challenges remaining are examined.
\end{abstract}

\section{KEYWORDS}

Lean Construction Management (LCM), Building Information Modelling (BIM), LCM Digital

\section{INTRODUCTION}

Achieving project cost, time and quality targets is extremely difficult. This is the result of increasing complexity, e.g. the demand for safety and (environmental) sustainability, and the increased number of project participants. As a result, construction projects are exposed to a vast range of challenges and feature top-down communication and a silo-oriented, linear mechanistic mindset. Conventional management systems are rather static and rigid, neglecting the fact that construction processes are stochastic with many unknown variables. They are often overwhelmed by unexpected events, and are thus often not accepted by the workforce. The underlying assumptions of conventional management approaches are that:

1 Managing Director, LCM Digital GmbH, Bundesallee 39-40a, 10717 Berlin, Germany, jakob.vonheyl@lcmdigital.com (www.lcmdigital.com)

2 Head of Lean-Design and -Construction, fischer Consulting GmbH, Klaus-Fischer-Straße 1, 72178 Waldachtal, Germany, selim-tugra.demir@ fischer.consulting (www.fischer.consulting) 
- Activities, tasks and dependencies can be defined in isolation

- Site workers and subcontractors share the same understanding of what needs to be done

- Required information is provided

- The desired outcome can be achieved on time if the plan / schedule is followed

However, this does not happen in practice. According to Demir and Theis (2018), projects that do not use any form of LCM approach show a Percent Plan Complete (PPC) of between $40 \%$ to $60 \%$. This means that many activities are not executed on time. Given this background, LCM and BIM have been accepted by the industry as the most promising methodological approaches to improving project reliability and performance (Sacks et al., 2009; Mollasalehi et al., 2018).

\section{AIM AND OBJECTIVES}

In the past, the two methodological approaches were normally used independently of each other (Sacks et al., 2010). BIM has been used for LCM to enable a better understanding of the object and the related interfaces. LCM, in turn, has been used for BIM as a simpler way of communicating product information to project participants. LCM and BIM do not depend on each other, i.e. LCM works with and without BIM, and the other way round (Sacks, 2009). However, a synthesises of the two methodologies allows the full potential to be realized, i.e. to achieve improved project productivity, resource utilization and delivery times (Singhal et al., 2018; Fosse et al., 2017; Tillmann \& Sargent, 2016). The combined use of LCM and modern technology has been the subject of several scientific studies and practical tests. It has been shown that the two approaches are mutually supportive (Fosse et al., 2017; Toledo et al., 2014; Dave et al., 2011). Sacks et al. (2009) and Koskela et al. (2010) as well as Sacks et al. (2010) explored 56 possible interactions between LCM and BIM. Based on this work, Mollasalehi et al. (2018) started the development of the LCM and BIM Maturity Model. So far, the solutions for possible interactions have been more theoretical. Recent studies, however, also analysed the possible interactions between LCM and BIM at trade contractor level (Tillmann \& Sargent, 2016; Murphy et al., 2018; Singhal et al., 2018). A study by Fosse et al. (2017) showed the results of the practical implementation of LCM and BIM by a Swedish contractor. The common feature of these studies is that - even though LCM and BIM are linked in a certain way to each other - there is still no true interaction. The focus is more on using synergies, deriving meaning, and visualisation. Given this background, there is still demand for an integrative platform to improve ongoing exchange of project, product and resource information. The aim of this research is to close this gap within the existing body of knowledge. In order to do so, this contribution presents the current development status of a platform called 'LCM Digital', the objectives of which are to:

- Improve collaborative work across trades, technologies and locations 
- Harmonize analogue and digital work processes

- Systematic compiling, preparation, analysis and assessment of product, project and process information (big data and machine learning)

- Ongoing evaluation of live data, forecasting

- Automatic project support through the provision of solution proposals using artificial intelligence

\section{THEORETICAL CONCEPT FOR INTERACTION BETWEEN LCM AND BIM}

The linking and integration of LCM, BIM and other tracking technologies (e.g. via Bluetooth low-energy sensors, QR codes and mobile devices) is very promising (von Heyl and Teizer, 2017; Cheng et al., 2010; Sacks et al., 2010). To date, BIM construction projects have usually only been modelled down to the level of detail of a master or phase schedule (Dave et al., 2011). The reason for this is that linking tasks, durations, dates and dependencies down to the lowest level is not part of the normal planning process, nor can automated linking currently be guaranteed (Dave et al., 2011). Furthermore, manual linking is very time-consuming. A great deal of research and development into suitable methods and software solutions to improve BIM- and LCM-based planning and scheduling processes is still required (Harris and Alves, 2013). Early alignment of LCM and BIM ensures stable interaction throughout the entire course of the project.

The interaction between LCM and BIM mainly involves the exchange of constructionrelated information. According to the ISO 12006-3, basic information about construction work can be categorized into products, processes and resources. The object-oriented product information is provided by the BIM model. Process information is the result of LCM planning and scheduling activities. Resource information can be retrieved from the systems that store supply chain information such as properties, capacities and availability of certain resources.

If BIM and LCM are successfully aligned, permanent interaction, exchange and adjustment of this information takes place during planning and execution to reduce variability and optimize workflow. The information, particularly in the central field of interaction, has to be combined and evaluated in such a way that relevant preconditions are considered (Ballard and Howell 2003). Koskela (2000) explores seven preconditions that avoid waste in site processes:

- Construction design (information)

- Components and materials

- Workers

- Equipment

- Space 
- Connecting works - previous work

- External conditions - such as weather, government regulations, licenses

The preconditions are named 'seven flows' (Koskela, 2000). If the seven flows are in place, an activity can start and finish without interruption or delay. This means that the seven flows can be permanently addressed if interaction between LCM and BIM is functioning well. This results in reliable and accepted data, which in turn leads to improvements in productivity, durations, and resource consumption and utilisation.

\section{PROPOSED FRAMEWORK}

The interaction between LCM and BIM during production planning and execution combines a top-down planning approach with a collaborative bottom-up evaluation of constructability. This engages the workforce in both approaches at the same time: A solution-oriented hands-on collaborative approach as well as a digitally planned and controlled production management approach. The outcomes are more realistic schedules based on 'hands-on' experience. The proposed framework - which basically consists of three LCM and BIM interactions - facilitates this.

First, production planning takes place. This is done in a range of workshops. The schedules are based on the geometric ground plans of the construction project as accessed via the BIM model. The platform also provides process templates for highly repetitive processes, and an activity list for the planning and assignment of tasks related to the planning and execution process. All information is stored centrally and exchanged as needed, getting as close to the vision of a single data source as possible.

Secondly, the data records generated during the planning process are brought to the construction site as work instructions, e.g. in the form of Kanban cards (digital or analogue - as required).

Thirdly, the platform facilitates the active management and control of process execution. The status of current processes and activities is updated semiautomatically using mobile apps and QR codes, enabling reliable determination of key performance indicators to support the early detection of problems, conflicts and constraints. This makes it possible to view and investigate basic system behaviour in a more targeted way, facilitating fundamental and cross-project improvements. The proposed framework for the integration of LCM and BIM is summarized in the Figure 2. 


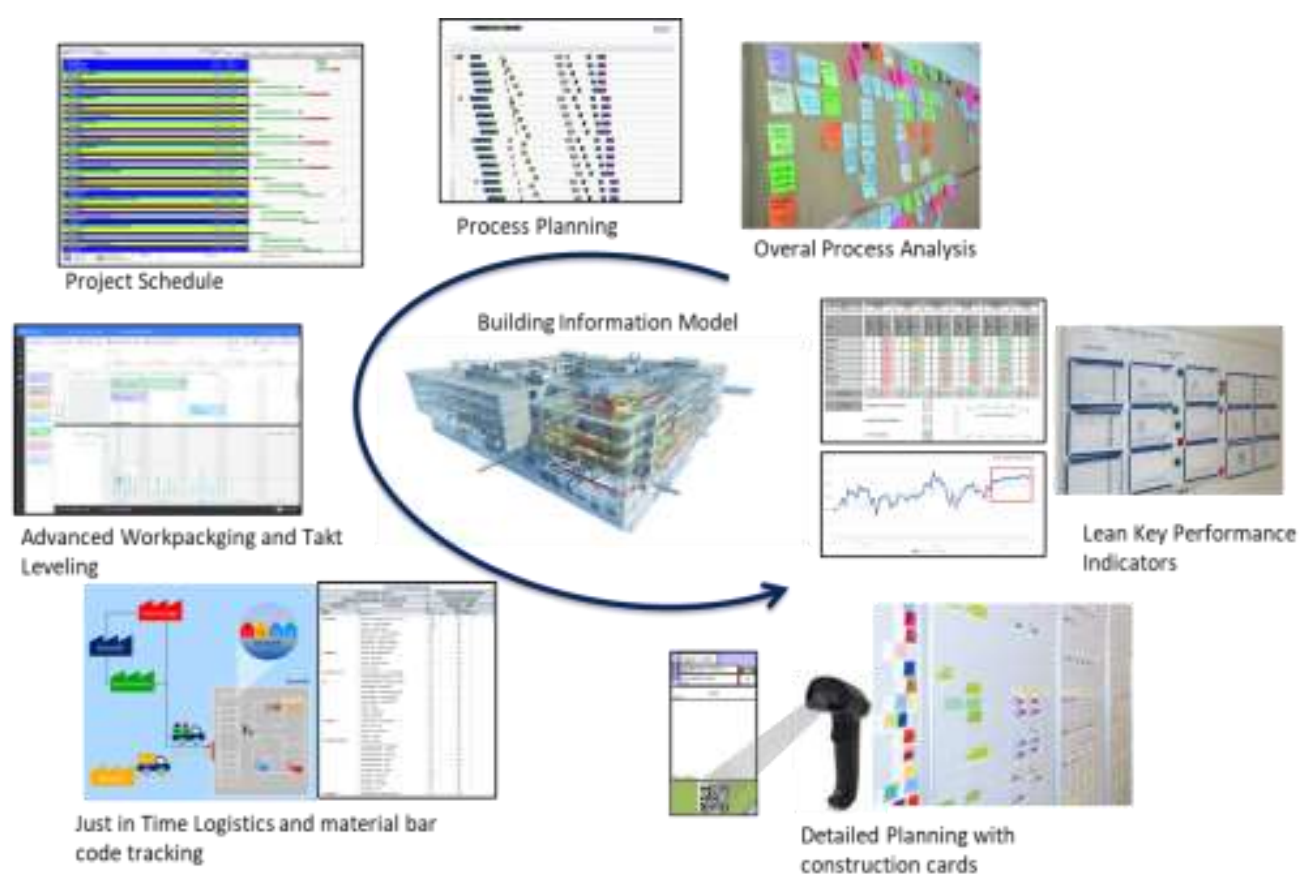

Figure 1: The proposed framework for Lean / BIM interaction

Figure 1 illustrates the proposed framework for the interaction between LCM and BIM, i.e. LCM Digital. The BIM model and the associated information is used to define the execution strategy and implement overall process analysis. This was undertaken in a pull planning workshop. The project schedule was further detailed through process planning. The seven flows (Koskela, 2000) are tracked in cyclical meetings during process planning workshops. The lookahead plan for process planning is between three to six months. The continuous review of preconditions according to the seven flows (Koskela, 2000) enables detection of problems so that they can be solved or alternative process sequences can be determined. Takt planning and line balancing allow resource usage to be optimized to improve performance on site, harmonizing the project heartbeat and creating laminar workflow. To translate this information and strategy to the construction site, daily Kanban cards (work instructions for each trade) are exported and printed out by the online platform. Each Kanban card is a daily target for a crew on site. The start and end of a task are identified with a QR code on the associated Kanban card. These are scanned with a smartphone application by the site team to track the status of the task. The moment the card is scanned, the task progress information is updated on the online platform. The BIM model is also updated, allowing visualization of tasks that have been started, are in progress and have been completed. This facilitates the daily detection of deviations and corresponding problems, which forms the basis for systematic improvement. Overall, this results in a transparent visual management system that is updated in real time and that increases site performance by promoting proactive behaviour. The following sections describe the main elements of the proposed framework in detail. 


\section{Production PlanNing WITH THE OVERALl PROCESS ANALYSIS, PROCESS PLANNING AND 4D BIM}

The central goal of BIM is to manage all project-related data in a virtual building. When the objects are linked to a schedule, the system is called 4D BIM. One common approach is to import the existing schedule into the BIM system. Even if scheduling is undertaken directly in the BIM software schedule application, there is no real change in the way the scheduling is approached. The underlying assumptions of the traditional conversation model remain the same:

- Top-down approach to manage major milestones

- Activities are prioritised by project and construction management

- Schedule content will be understood by the contractors

- Activities can be scheduled precisely

- Activities will start and finish on schedule

Unfortunately, most of these assumptions do not usually apply. 4D BIM can provide many benefits, such as a better understanding through visualization of structural conditions and object-oriented simulation of the schedule. However, if the social management system remains inadequate, an improved technical management system - even a BIM-supported one - cannot truly benefit the project. The conventional conversation model and the related social system can be improved through the integration of hands-on and collaborative workshops. In the ideal case, overall process analysis is undertaken prior to the start of the project. The 3D BIM model supports the workshop through proper visualisation of the project. The workshop is conducted with all available parties. The overall process analysis optimises the entire project process for repetitive and non-repetitive areas. The process steps are sequenced using pull planning, from the back to the front. This is done on brown paper with sticky notes. Remaining design activities are also integrated into the overall process analysis workshops. Bottleneck activities and trades are identified as part of an overall process analysis workshop. Problems, risks and action points are assessed and documented. The results are digitized and then form the basis for the identification of the takt areas. Once the takt time and takt area are clear, the takt train is defined. Metaphorically, the takt train travels through the different takt areas of the building sequencing the various activities accordingly. The number of takt trains running at the same time needs to be clarified based on project circumstances, i.e. resources and requirements.

Process planning integrates the taktable and non-taktable areas. This is done in handson workshops. This is undertaken for the entire project duration prior to the start of the project. Lookahead planning is then undertaken in cyclical workshops. The goal of these process planning workshops is to update the activities for the upcoming three to six months. The seven flows (Koskela 2000) are also tracked to enable an activity to start and finish. The results of the workshop are digitized and uploaded to the 4D BIM. This means that depending on the size and complexity of the project - process planning workshops can be purely digital. 


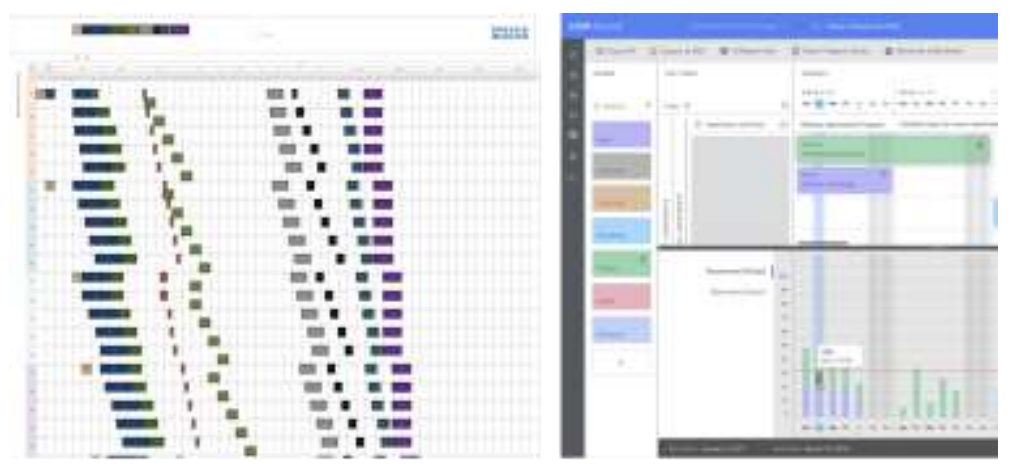

Figure 2: Print view of lookahead planning (left) and platform's data entry field

Figure 2 shows the input and output of LCM Digital. All relevant information regarding duration, quantity, sequence, availability, reliability was tracked through the cyclical interaction between 4D BIM and process planning workshops. The outcome was a more accurate and reliable production planning system.

\section{PRODUCTION CONTROL AND CONTINUOUS PROCESS IMPROVEMENT WITH THE LAST PLANNER AT THE CONSTRUCTION SITE AND BIM}

A planning board for the Kanban-Cards was prepared and adapted to the requirements of the construction site. Each row represents one repetitive or non-repetitive work or takt area. Each column represents one day. For the first three to four weeks, each trade details their activities at the planning board daily. Each activity - comprising several predefined operations or daily tasks - is linked to the BIM model by an activity ID. Figure 3 shows how the selected element within the BIM model is related to an activity by an activity ID. Activities that need to be executed are selected in the BIM model. The selection results in a colour coding. Figure 3 shows how the elements selected within the BIM model are shown red. Project participants make selections manually based on process planning.

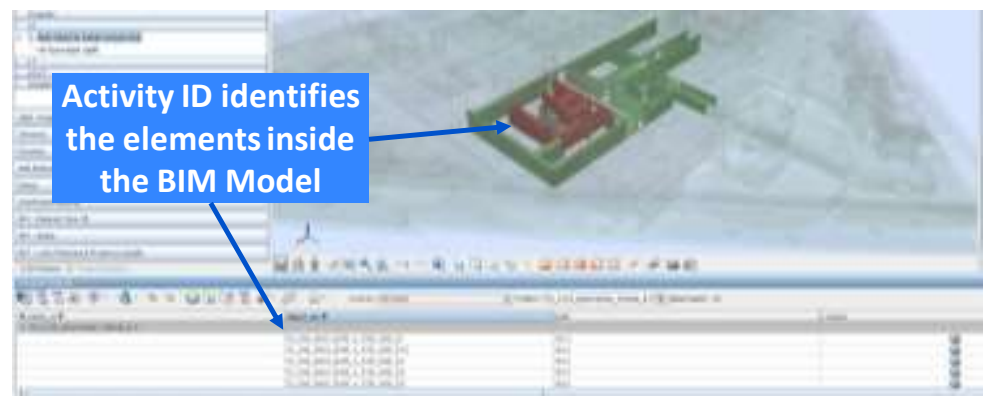

Figure 3: Linking activities and BIM-objects

The information stored in the model also supports prioritisation, e.g. the duration, sequence, and the seven flows (Koskela 2010). Kanban cards are printed by the BIM-linked online platform based on this prioritisation. 


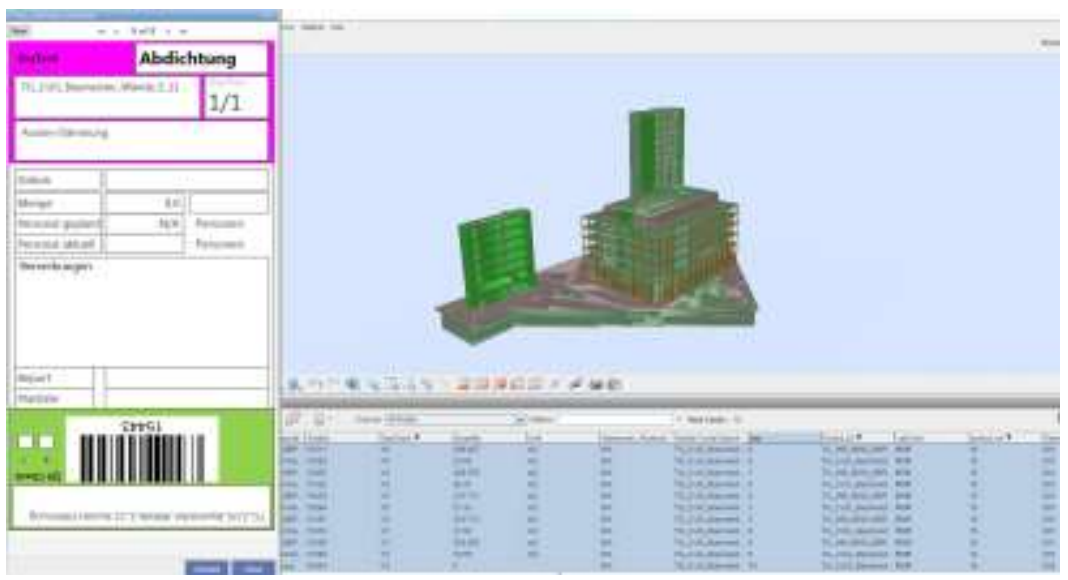

Figure 4: Generating Kanban cards for the last planner at the construction site

Figure 4 shows the printed Kanban cards, which include a QR code to link the virtual and real worlds. Progress is updated and monitored during daily lean leader standard work or lean routines at the construction site, such as daily meetings. The Kanban cards represent the targets for the day. Target deviations are tracked daily. Reasons for deviations are identified and analysed, allowing improvements to be made. Kanban cards relating to started or finished processes are scanned daily and the systems updated. The result is a live status and progress update. Completed cards can be double-checked during daily lean waste walks to ensure that data sets are current and correct prior to any further action.
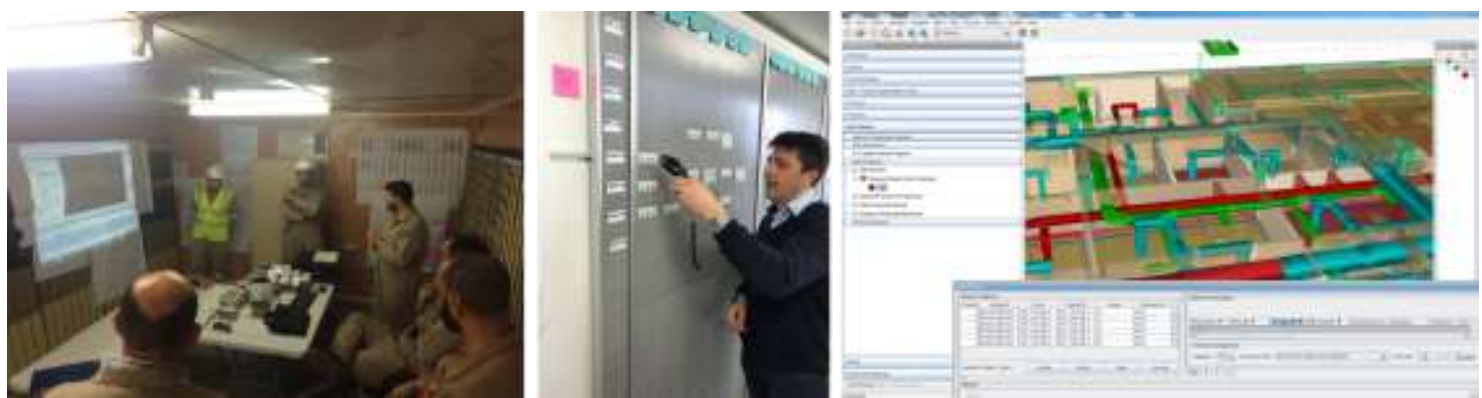

Figure 5: Impressions of the interaction between LCM and BIM at the construction site

Figure 5 shows the interaction between the virtual BIM model and the real construction site. This interaction is primarily enabled through the Kanban cards, tagged with a QR code and printed from the platform. This interaction allows the relevant individuals to gather more accurate information in a timely manner to improve performance. The conventional conversation model is replaced by a new collaborative conversation and interaction model. The project can benefit from knowledge and experience at all levels. Buffers, contingencies, delays and idle time are visualized in real time, supporting the continuous improvement of the construction processes. 


\section{CONCLUSION}

It has been demonstrated that a platform that facilitates the exchange of information related to products, processes and resources is a key success factor for the integration of LCM and BIM into projects. A successful alignment of LCM and BIM, as well as systematic evaluation of collected data, contributes decisively to an improvement of the general project management as well as to the success of a specific project. So far, several technological concepts have been developed and tested. These include:

- Automated printing of construction assignments (Kanban cards)

- Digital pull planning workshops

- Use of BIM-based quantity take-offs for accurate resource planning and reliable assessment of project performance based on live data (mobile app and QR coding technologies)

One of the main current challenges is the uniform structuring and automated linking of execution-related data sets down to individual elements of a BIM model to facilitate collaborative project execution. Only the acceptance and systematic use of the platform by the workforce will allow meaningful data to be generated. So far, user feedback has been quite positive, stating that it is hands-on, easy and flexible. In conclusion, it can be stated that systematic linking of BIM and LCM via a central platform, as outlined in this paper, validates information and makes it available in a timley and targeted manner. It promotes the cooperation and communication between the individual project participants, and thus makes a positive contribution to social management.

The findings were processed and incorporated into the programming of a web-based software solution called LCM Digital. Further research and development activities will focus on exploring the integration of artificial intelligence, big data and user-experience concepts.

\section{REFERENCES}

Ballard, G. and Howell, G. (2003). Lean project management. Building Research \& Information, 31:2, 119-133.

Cheng, T., Yang, J., Teizer, J, and Vela, P.A. (2010). Automated Construction Resource Location Tracking to Support the Analysis of Lean Principles, Proc. 18th Ann. Conf. of the International Group for Lean Construction, Haifa, 643-653.

Dave, B., Boddy, S. and Koskela, L. (2011). Visilean: Designing a production management system with Lean and BIM. 19th Annual Conf. of the International Group for Lean Construction (IGLC-19), Lima.

Demir, Selim-Tugra and Theis, Patrick (2018). Die Interaktion von Building Information Modeling und Lean Construction Management. Bauingenieur Magazin, 98:2018 (annual issue), 130-135. 
Fosse, R., Ballard, G. and Fischer, M. (2017). Virtual Design and Construction: Aligning BIM and Lean in Practice. Proc. Lean \& Computing in Construction Congress $\left(L C^{3}\right)$, Vol. 1 (CIB W78), Heraklion, 499-506

Gerber, D, Becerik-Gerber, B. and Kunz, A. (2010). Building Information Modeling and Lean Construction: Technology, Methodology and Advances from Practice. 18th Annual Conf. of the International Group for Lean Construction (IGLC-18). Haifa, 683693

Harris, B. and Alves, T. (2013). 4D Building Information Modeling and Field Operations: An Exploratory Study. 21st Annual Conf. of the International Group for Lean Construction (IGLC-21), Fortaleza, 811-820

Koskela, L. J. (2000). An Exploration Towards a Production Theory and its Application to Construction. Espoo: VTT Technical Research Centre of Finland.

Koskela, L., Owen, B., \& Bhargav, D. (2010). Lean Construction, Building Information Modelling and Sustainability. ERACOBUILD Workshop on BIM and Lean, Malmö: Queensland University of Technology,1-9.

Mollasalehi, S., Aboumoemen, A. A., Rathnayake, A., Fleming, A., \& Underwood, J. (2018). Development of an Integrated BIM and Lean Maturity Model. 26th Annual Conf. of the International Group for Lean (IGLC-26), Chennai, 1217-1228

Murphy, Z., Saripally, D., Dhakla, S., Trujillo, R., Luttmann, E., \& Sreekumar, A. (2018). Leveraging advanced VDC methods and reality capture to increase the predictability for prefabrication. 26th Annual Conf. of the International Group for Lean (IGLC-26), Chennai, 101-111

Sacks, R., Dave, B., Koskela, L., \& Owen, R. (2009). Analysis Framework for the Interaction Between Lean Construction and Building Information Modelling. 17th Annual Conf. of the International Group for Lean (IGLC-17), Taipei, 1-10

Sacks, R., Radosavljevic, M., and Barak, R. (2010). Requirements for building information modeling based lean production management systems for construction, Automation in Construction, 19(6), 641-655

Singhal, N., Ahuja, \& Ritu. (2018). can BIM furnish Lean benefits - an Indian case study. 26th Annual Conf. of the International Group for Lean (IGLC-26), Chennai, 90-100

Tillmann, P., \& Sargent, Z. (2016). Last Planner \& BIM Integration: Lessons from a Continuous Improvement Effort. 24th Annual Conf. of the International Group for Lean (IGLC-246), Boston, 113-122

Toledo, M., González, V. A. , Villegas, A. and Mourgues, C.(2014). Using 4D Models for Tracking Project Progress and Visualizing the Owner's Constraints in Fast-Track Retail Renovation Projects. 22nd Annual Conf. of the International Group for Lean Construction (IGLC-22), Oslo, 969-980

von Heyl, J. and Teizer, J. (2017). Lean Production Controlling and Progress Tracking using Digital Methods. Proc. Lean \& Computing in Construction Congress $\left(L C^{3}\right)$, Vol. 1 (CIB W78), Heraklion, 127-134 